\title{
Paludisme, institutions et croissance : que penser du débat actuel?
}

Daniel Longuépée

\section{(2) OpenEdition \\ 1 Journals}

Édition électronique

URL : http://journals.openedition.org/ei/1125

DOI : 10.4000/ei. 1125

ISSN : 2553-1891

Éditeur

Association Économie et Institutions

Édition imprimée

Date de publication : 1 juin 2006

Pagination : 95-118

ISSN : 1775-2329

\section{Référence électronique}

Daniel Longuépée, «Paludisme, institutions et croissance : que penser du débat actuel ? ", Économie et institutions [En ligne], 8| 2006, mis en ligne le 31 janvier 2013, consulté le 10 décembre 2020. URL :

http://journals.openedition.org/ei/1125 ; DOI : https://doi.org/10.4000/ei.1125

Revue Économie et institutions 


\section{Paludisme, institutions et croissance : que penser du débat actuel ?}

\section{Daniel Longuépée ${ }^{1}$}

L'intérêt pour l'analyse des relations entre environnement géographique et environnement institutionnel est loin d'être récent. Il suffit d'évoquer la théorie des climats dont Montesquieu, au XVIII siècle, constitue l'une des figures emblématiques. Loin de se réduire à un déterminisme qui condamnerait pour toujours les peuples à la pauvreté, cette théorie cherche plutôt à retrouver de l'ordre derrière la diversité des mœurs, des façons de gouverner observées à l'étranger, etc. ${ }^{2}$. Certes le climat agit directement selon Montesquieu sur le tempérament et le caractère des individus. Mais si la disposition au travail par exemple est liée au climat, des mesures spécifiques de nature institutionnelle peuvent la modifier. L'intitulé du chapitre V du livre XIV de L'Esprit des lois est à ce titre révélateur : "Que les mauvais législateurs sont ceux qui ont favorisé les vices du climat, et les bons sont ceux qui s'y sont opposés ". Il est ainsi toujours possible d'orienter différemment les voies tracées par la nature ${ }^{3}$. En ce sens il doit exister une relation intime entre

1 CRIISEA, Université de Picardie.

2 "Aussi fragmentaire et confuse qu'elle ait été alors, l'incessante collecte de détails sur les mœurs et coutumes des différents peuples de la terre dévoile aux yeux des Européens l'infinie diversité des langues et des cultures mais aussi des différences considérables entre les organisations sociales. Aussi est-il probable qu'émerge dès cette époque le paradoxe qui restera si longtemps au cœur des débats sur les origines et qui freinera la pensée anthropologique au XVIII siècle: comment inscrire la diversité de la nature humaine dans un discours rationnel, tout en maintenant son unité fondamentale. On comprend l'attrait qu'exerce alors la théorie des climats dans l'élaboration de systèmes philosophiques et même scientifiques qui cherchent une cohérence interne dans le rapport des hommes à leur environnement : la différence de latitude explique celle des mœurs et par conséquent les écarts entre institutions ou gouvernements. Nature, culture et société peuvent être pensées à l'intérieur d'une dynamique préfixée mais dialectique, et non dissociées dans un chaos comme le constat de diversité aurait pu le laisser craindre ", Nicole Hafid-Martin (1999).

3 Le caractère particulièrement nuancé de l'argumentation de Montesquieu est souligné par Chevallier (1993) et Claval (2003). Ce dernier qualifie la théorie des climats de Montesquieu d'" environnementalisme hippocratique ", (p. 68 et s. ). Il s'agit de voir quelle est l'influence du milieu géographique sur les humeurs, tout en reconnaissant la nature variable de cette influence. En ce sens il n'y a pas de causalité stricte. L'explication est à la fois physiologique et psychologique. Elle paraît en tout cas beaucoup plus 
géographie et institutions. Montesquieu s'appuie entre autres sur le cas de la Chine. "Les législateurs de la Chine furent plus sensés lorsque, considérant les hommes, non pas dans l'état paisible où ils seront quelque jour, mais dans l'action propre à leur faire remplir les devoirs de la vie, ils firent leur religion, leur philosophie et leurs lois toutes pratiques. Plus les causes physiques portent les hommes au repos, plus les causes morales les en doivent éloigner ", (Montesquieu, 1979 [1748], p. 379). Nous pouvons ici associer les causes "morales" aux institutions informelles au sens de North (1990) c'est-à-dire à l'ensemble des habitudes, des coutumes, etc. Nous aboutissons alors moins à une théorie des climats qu'à une théorie de la compensation des facteurs (de la croissance).

Les réflexions de Montesquieu nous paraissent intéressantes dans la mesure où elles éclairent le débat mené depuis quelques temps en grande partie par des économistes américains sur les causes profondes de la croissance et du développement. Les institutions jouent un rôle central dans la mesure où elles peuvent modifier la trajectoire économique d'un pays. C'est notamment le sens de la démarche initiée par Acemoglu, Johnson et Robinson (2002), qui considèrent que la bonne ou mauvaise "fortune" d'un pays peut être inversée 4 . Ainsi les pays initialement riches ont été appauvris par de mauvaises institutions et les pays initialement pauvres enrichis par de bonnes institutions. Les auteurs suggèrent, faut-il croire, un principe implicite de compensation, à la Montesquieu, où "[n]i la géographie ni l'histoire, par exemple, ne "prédestinent " un pays à avoir de "bonnes " ou de "mauvaises institutions ", (Fonds monétaire international, 2003, p. 111). La cause semble ici entendue. Certes les pays peuvent faire face à un mauvais climat, une mauvaise situation géographique mais il est possible de compenser ce handicap initial par de bonnes lois ou encore des règles de vie spécifiques, bref par un cadre institutionnel approprié. Ce raisonnement bien que plausible est toutefois contesté par un groupe d'auteurs autour de Sachs, qui considèrent qu'en face

prudente que celle défendue par 1' " environnementalisme évolutionniste ", qui va jusqu'à effectuer un lien strict entre réussite et environnement, voir à établir une typologie des races. Même Ratzel, l'un des inspirateurs de ce courant, finit par adopter une position moins sectaire en distinguant peuple de nature et peuple de culture. Il rompt ainsi avec une causalité géographique trop stricte. "En opposant les peuples de nature et les peuples de culture, il montre que l'influence du milieu cesse d'être aussi déterminante dès l'instant où les hommes ont appris à se structurer -à se doter, en particulier, de l'Etat, l'instrument essentiel qui leur permet d'organiser l'espace à leur profit ", (p. 73).

${ }^{4}$ D'où l'intitulé de l'une de leur contribution: "Reversal of Fortune: Geography and Institutions in the Making of the Modern World Income Distribution ".

96 Economie et Institutions $-\mathrm{n}^{\circ} 8-1^{\mathrm{er}}$ semestre 2006 
du fléau géographique que constitue le paludisme, les réformes institutionnelles sont inopérantes. En ce sens il n'y a pas de compensation possible du type: des institutions adéquates compenseraient un handicap initial lié à l'existence d'une maladie tropicale. La compensation doit se faire à un autre niveau, celui de l'aide internationale par exemple. Sachs (2003, p. 38) déclare notamment que les pays pauvres ont "besoin de bien plus que de discours sur la bonne gouvernance" ou sur les "bonnes institutions". Ils ont besoin d'interventions directes, soutenues par une aide accrue des bailleurs de fonds (...)" ".

Cependant, si nous suivons effectivement l'exemple du paludisme, devons-nous considérer qu'il constitue une cause entièrement naturelle ? N'a-t-il pas une nature institutionnelle liée à sa dimension socioculturelle ? Par exemple la distribution gratuite de moustiquaires, comme le prône Sachs (2006), sera-t-elle suffisante sans prise en compte des modes de vie et des représentations collectives? Par ailleurs amoindrir le rôle des institutions revient à nier les travaux qui ont précisément mis l'accent sur leur place décisive. Or doit-il y avoir concurrence entre une explication qui serait "paludique " et une autre qui serait "institutionnelle"? Ou ne faut-il pas plutôt viser uniquement le discours sur la "bonne gouvernance ", qui renvoie à une conception particulière des institutions ? Répondre à ces questions suppose de réfléchir à la manière d'envisager le cadre institutionnel et le paludisme mais aussi à la représentation de l'action de chacune de ces deux variables. En cherchant les causes profondes de la croissance les auteurs oublieraient d'une manière générale les interactions fécondes entre les variables.

Aussi nous allons nous intéresser d'abord à la nature véritable du paludisme. A partir de là nous pourrons aborder les termes actuels du débat et montrer dans quelle mesure ils apparaissent en partie tronqués.

\section{Le paludisme, une maladie pluridimensionnelle.}

Puisque le paludisme, comme maladie tropicale, sert à justifier certains retards de développement, il convient dans un premier temps d'en évaluer la nature profonde. S'agit-il vraiment d'un fléau multiforme? Sa diffusion est-elle liée à un statut entomologique ou repose-t-elle aussi sur un environnement socioéconomique spécifique ? Après avoir abordé les spécificités " naturelles " de la maladie, nous en évaluerons la dimension socioculturelle. 


\section{1. Vecteurs de transmission du paludisme, capacité de résistance des moustiques et immunité.}

Le paludisme est une maladie très ancienne qui apparaît avec les hommes ${ }^{5}$. Il semblerait que les ancêtres d'Homo sapiens souffraient déjà de fièvres caractéristiques en relation étroite avec les eaux stagnantes des étangs et marais (Josserand Debacker, 2000). Malgré les programmes de lutte mis en place, le paludisme reste aujourd'hui un fléau de tout premier ordre. "Le paludisme tue un enfant toutes les 30 secondes en Afrique et entre 1 et 3 millions de personnes par an, selon les estimations de l'OMS. Deux milliards d'individus, soit $40 \%$ de la population mondiale, sont exposés et on estime à 500 millions le nombre de cas cliniques survenant chaque année... Le paludisme touche une centaine de pays dans le monde, particulièrement les zones tropicales défavorisées d'Afrique, d'Asie et d'Amérique Latine. L'Afrique est, de loin, le continent le plus touché avec $90 \%$ des cas de paludisme recensés dans ses zones tropicales ", (Institut Pasteur, 2005) ${ }^{6}$.

Le paludisme est transmis par les moustiques du genre " anophèle " dans le cadre de ce qu'on appelle le cycle du plasmodium. Ce cycle comprend deux phases essentielles : un cycle asexué chez l'homme, qui touche indifféremment les hommes et les femmes et un cycle sexué chez le moustique où seules les femelles sont concernées. Le moustique pique d'abord un individu et lui injecte le parasite. Ce dernier se développe sous une autre forme jusqu'à une nouvelle piqûre qui entraînera alors le développement du parasite chez le moustique, via son tube digestif, et ainsi de suite. En tout cas si les moustiques sont répandus dans le monde entier, il n'y a qu'une vingtaine d'espèces dangereuses, notamment la plus résistante, l'Anophèles gambiae, présente uniquement en Afrique subsaharienne ${ }^{7}$.

5 Remarquons que le terme "paludisme " correspond au terme anglo-saxon " malaria ".

${ }^{6}$ La prise de conscience du fléau que représente le paludisme est mondiale. Il suffit de citer le partenariat "Faire reculer le paludisme" (Rollback Malaria), créé en 1998 par l'OMS, l'Unicef, le Programme des Nations Unies pour le Développement et la Banque mondiale. "Faire reculer le paludisme" regroupe les gouvernements des pays affectés par la maladie, des bailleurs de fonds, des ONG, des instituts de recherches et des universités. Des sommets mondiaux sont aussi organisés. Par exemple, les réflexions issues de la conférence tenue en Tanzanie en novembre 2002 ont été l'occasion d'un numéro spécial de la revue American Journal of Tropical Medecine publié en 2004 (Supplément au volume 71).

7 D'une manière générale, l'anophèle est un " insecte piqueur, hématophage ; rural, il affectionne les régions chaudes et humides, aime peu l'altitude, et sa distance de vol ne dépasse pas un à deux kilomètres ", (Duriez et Golvan,

98 Economie et Institutions $-\mathrm{n}^{\circ} 8-1^{\mathrm{er}}$ semestre 2006 
A côté de cette particularité spatiale, se dessine une évolution préoccupante concernant la capacité de résistance des moustiques, notamment aux DTT, pesticides qui ont été pourtant efficaces selon Duriez et Golvan (1996). La résistance des moustiques pose en soi de graves problèmes puisqu'il faut renouveler les médicaments permettant de lutter contre le paludisme, notamment les plus utilisés comme la chloroquine et la sulphadoxinepyrimethamine (Brun et al., 2004). L'idée selon laquelle la nature est capable de se protéger renforce le statut du paludisme. Il est prouvé par exemple que les antibiotiques perdent à la longue de leur efficacité.

Il semble de toute façon que le risque zéro soit impossible en matière épidémiologique d'une façon générale. "Le risque épidémique et sa perception seront toujours présents dans nos sociétés pour des raisons psychologiques car nous cultivons le culte du risque zéro, tout en aspirant à un mode de vie, par exemple aux voyages touristiques lointains, facteurs de risques ; d'autre part, si le développement économique permet d'éradiquer certains risques infectieux, il en génère d'autres. C'est un fait, notre environnement est peuplé de micro-organismes : il ne sera jamais stérile, ce n'est ni possible, ni souhaitable", (Office parlementaire d'évaluation des choix scientifiques et technologiques, 2005, tome 1, p.12).

En opposition à la capacité de résistance des vecteurs de transmission, nous devons évoquer la constitution d'une immunité chez les individus, pouvant éventuellement contrebalancer la force du paludisme. Une telle immunité est en effet possible à travers ce qu'on appelle le trait drépanocytaire, touchant essentiellement la population noire. Ce point est important car il sert justement d'argument à Acemoglu, Johnson et Robinson (2001) pour minimiser le statut du paludisme. Le problème cependant c'est que ce trait transmis par les deux parents à l'enfant peut se transformer en anémie à cellules falciformes conduisant alors à une mort prématurée quasi certaine. Carstensen et Gundlach (2005) ont alors beau jeu de rappeler la possible dégénérescence en cas de double transmission, ce qui invalide l'idée d'une immunité protectrice à la longue.

Nous pouvons dans ces conditions comprendre la démarche de Sachs et de ses collaborateurs cherchant à évaluer le coût du paludisme à travers ses conséquences sur l'activité économique. "Si certains peuvent s'étonner de la capacité d'une maladie à paralyser le développement économique, c'est qu'ils ne comprennent pas bien comment une maladie peut influer sur les résultats économiques ",

1996, p. 418). Si nous ajoutons le climat chaud et humide avec de faibles variations, nous comprenons pourquoi il existe les conditions d'une "transmission stable et généralement intense du paludisme ", (Carter et Mendis, 2002, p. 568). 
(Sachs, 2003, p. 39) ${ }^{8}$. Or ce ne sont apparemment pas les occasions d'influence qui manquent : maintien d'un taux élevé de natalité afin de compenser les pertes à venir d'enfants, peu d'incitation à investir dans l'éducation des enfants si ceux-ci doivent mourir, conséquences directes sur les capacités physiques et mentales de la population (déficiences irréversibles, absentéisme scolaire, etc.), (Gallup et Sachs, 2001, Malaney, Sachs et Spielman, 2004, Breman, Alilio et Mills, 2004).

Le paludisme initie ainsi un cercle vicieux de la pauvreté. Hamoudi et Sachs (1999) montrent par exemple que les spécificités des vecteurs de transmission expliquent les succès et les échecs rencontrés dans les politiques de lutte contre le paludisme. Ainsi l'éradication du paludisme dans les pays européens au cours du siècle dernier, a laissé croire à une victoire de la croissance économique. En accédant à la croissance et au développement les pays dégageraient les ressources suffisantes pour éradiquer une maladie. Cependant, le cas européen n'est pas universel. En effet, la spécificité du climat et des vecteurs de transmission dans une zone à climat tempéré ne pouvait que favoriser l'éradication du paludisme. Dans ce genre de zones les vecteurs africains comme l'Anopheles gambiae particulièrement anthropophiles ne sont pas présents. Par ailleurs, et à titre d'illustration, les symptômes ont été davantage repérables car on ne pouvait pas les confondre avec d'autres maladies ; de même la sensibilité à l'irrigation est absente dans les zones tempérées. A côté du "succès" européen, il est tentant d'évoquer l'échec de Gorki au Nigéria. Gallup et Sachs (2001) notent que le gouvernement nigérien a notamment dépensé plus de 6 millions de dollars pour éradiquer le paludisme, sans résultat probant. En effet la réduction drastique du taux de piqûres par les moustiques ne s'est pas accompagnée d'une réduction du taux d'infection'.

L'éradication du paludisme ne serait donc nullement liée à une croissance initiale ou à un investissement particulier mais à la spécificité géographique des moustiques: les plus résistants sont localisés dans des zones climatiques spécifiques. Plus généralement pour Hamoudi et Sachs (1999), l'histoire épidémiologique du paludisme confirme le statut du paludisme. Dans la mesure où les symptômes de la maladie sont connus depuis l'Antiquité, les activités

8 "Il n'est pas exagéré de dire que là où le paludisme est présent, nous devons nous attendre à ce qu'il affecte la plupart des caractéristiques humaines incluant la mobilité, les choix d'investissement et même les décisions concernant la natalité ", (Malaney, 2003, p. 2).

9 "Les efforts de contrôle furent vaincus par la capacité vectorielle des moustiques qui dépassaient ce qui était requis pour maintenir la transmission du paludisme ", (Gallup et Sachs, 2001, p. 89).

100 Economie et Institutions $-\mathrm{n}^{\circ} 8-1^{\mathrm{er}}$ semestre 2006 
économiques ont au mieux accéléré le développement d'une maladie qui était de toute façon inévitable.

L'analyse conduit par conséquent à penser que le statut médical du paludisme est tel, que l'éradication de la maladie constitue la condition nécessaire et suffisante du développement dans certains pays. Est-ce toutefois aussi probant ? En insistant sur les conséquences économiques de la maladie, les auteurs ne passent-ils pas sous silence la dimension socioculturelle?

\section{2. La dimension socioculturelle du paludisme.}

Comme nous l'avons vu précédemment il existe un lien évident entre le paludisme et les moustiques qui sont les vecteurs de transmission. Toutefois s'arrêter à ce niveau n'est pas suffisant. Il semble en effet que les éléments culturels liés à la perception de la maladie en définissent largement la nature et l'étendue mesurable. Il existe alors des interactions complexes entre paludisme, écosystème et développement.

Si le cycle du paludisme est scientifiquement établi, il ne faut pas sous-estimer la manière dont les populations africaines se représentent le paludisme. Une telle analyse relève alors de l'anthropologie médicale qui "repose sur le postulat que la Maladie (fait universel) est gérée et traitée suivant des modalités différentes selon les sociétés et que ces modalités sont liées à des systèmes de croyances et de représentations déterminés, en fonction de la culture dans laquelle elle émerge ", (Fainzang, 2000).

Plusieurs éléments peuvent être mis en avant :

- Les populations ne font pas toujours le lien entre moustiques et transmission de la maladie. Cela entraîne par exemple une sous-utilisation des moustiquaires imprégnées d'insecticide (MII), pourtant efficaces. Les individus cherchent en effet avant tout à se protéger non pas tant du paludisme que de la gêne occasionnée par les piqûres (Audibert, 2004, Zimicki, 1997). De plus les populations se servent des moustiquaires lorsque le taux de moustiques est important (selon les saisons); or le paludisme se transmet aussi avec peu de moustiques. Ces comportements liés à des représentations particulières vont avoir des conséquences importantes en termes de politiques à mener. Zimicki (1997) se demande même s'il faut insister sur la réduction de la maladie ou la réduction de l'inconfort pour inciter les individus à utiliser des moustiquaires. Même si le motif d'inconfort prédomine au sein de la population (et peut donc jouer comme argument), en cas d'arbitrages financiers, les individus préféreront acheter du tabac plutôt qu'une moustiquaire. Les enfants peuvent aussi être les premières victimes des représentations puisque certaines communautés pensent qu'ils dorment plus profondément que les adultes et qu'ils sont donc moins 
gênés par les moustiques. Ces remarques sont importantes car elles conduisent à relativiser l'idée de moustiquaires comme panacée à la lutte antipaludique, idée que défend Sachs.

- Les symptômes du paludisme sont parfois confondus avec des maladies expliquées par les mauvais esprits ou la malchance. C'est notamment le cas pour les formes graves de paludisme. Il est alors considéré que les accès palustres engagent la communauté dans son ensemble à travers quelques individus. Les esprits visent une communauté donnée en lui envoyant un signe. Les individus doivent dans ce cas se soumettre à certaines exigences, et notamment aller voir en priorité un guérisseur local. Contourner ce passage, c'est s'exposer à l'ostracisme en cas de décès d'un enfant par exemple. Les cas de paludisme bénins sont par contre considérés comme des maladies mais parfaitement tolérables. Contrairement aux cas de sida ou de tuberculose, les symptômes du paludisme bénin ne sont pas stigmatisés. Par conséquent, la pression sociale n'incite guère à la recherche d'un traitement spécifique (Jones et Williams, 2004).

Par ailleurs les populations peuvent préférer un traitement local si la médecine occidentale ne donne pas les résultats escomptés. Le fait qu'une séance d'exorcisme permette de sauver une patiente atteinte de fièvre, non guérie par la médecine occidentale, renforce les doutes sur l'explication scientifique (Elverdan et Oberländer, 2000).

- Le système de paiement est aussi important dans la mesure où les facilités offertes par un guérisseur local favorisent le recours à la médecine locale (Hausman-Muela, Mushi et Ribera, 2000).

- Les structures médicales peuvent être sous-utilisées malgré une augmentation de l'offre de soins. C'est ce que montrent Audibert et ses collaborateurs (Audibert et al. 2001, 2004) dans le cas de nombreux pays sub-sahariens. Ce ne sont alors pas tant les capacités financières qui sont en cause que les habitudes de comportement. " [1]a différence de comportement face aux épisodes morbides entre les habitants de la forêt et ceux de la savane (avec un recours plus fréquent aux structures de soins modernes en zone de forêt qu'en zone de savane) semble plus lié à un comportement qu'à une justification économique : le niveau de vie moyen des habitants de la forêt est plutôt inférieur actuellement à celui des habitants de la savane " (Audibert et al., 2001, p. 24).

- Les structures sociales jouent enfin un rôle important dans la séquence de guérison même dans le cas où le rôle du moustique est convenablement interprété. Ainsi les structures patriarcales, où les hommes sont responsables des enfants et disposent de l'argent peuvent retarder l'administration de traitement lorsque les femmes sont seules avec leurs enfants (Elverdan et Oberländer, 2000). D'ailleurs les femmes ont un rôle social qui les défavorise nettement 
dans l'accès aux soins. Une telle idée renvoie à la notion plus large de "vulnérabilité sociale ". "Dans chaque société, il y a des groupes d'individus qui ont, à cause de rôles socialement et culturellement définis, un contrôle limité sur leur habilité à admettre une maladie, à mobiliser des ressources, à accéder à des services et à prendre des décisions ", (Jones et Williams, 2004, p. 158). Plusieurs variables discriminantes peuvent entrer en ligne de compte: l'âge, le sexe, l'ethnie, l'affiliation politique, le statut socioéconomique, etc. Il existe d'une manière générale un lien entre le statut socioéconomique d'une personne et son degré d'exposition à la maladie, et au paludisme en particulier.

Nous voyons donc qu'il n'est pas aisé de savoir ce qui doit être effectivement mesuré lorsque nous parlons du paludisme. Être malade n'est pas socialement neutre. Ainsi l'observation du fléau que constitue le paludisme ne reposera pas uniquement sur l'endémie de la maladie, le statut immunologique et la santé sous-jacente mais aussi sur l'organisation sociale et le statut des croyances culturelles. La " perception de la maladie interagit avec la réalité socioculturelle des individus (leur rôle social et les comportements attendus) pour structurer à la fois leur comportement et leur capacité à répondre à la maladie ", (Jones et Williams, 2004, p. 158).

Il semble bien qu'une analyse centrée sur la relation moustique (vecteur de transmission)-paludisme demeure incomplète dans la mesure où elle ne permet pas de voir justement la nature complexe du paludisme. Interprétant l'approche en termes de santé écosystémique, cherchant à intégrer toutes les interdépendances, Pépall (2003, p.2) déclare que nous pouvons "comparer cette approche à un appareil photo avec zoom. Le zoom montre de près les détails du problème-dans le cas du paludisme, un moustique qui transmet le parasite à quelqu'un. Lorsqu'on éloigne l'objectif, d'autres éléments apparaissent : la pauvreté dans les villages; les pratiques culturales dans les rizières; des hommes qui restent assis à ne rien faire tandis que leurs femmes travaillent. Bref, cette perspective " grand-angulaire " aide les chercheurs à déterminer les motifs que cachent les statistiques sur le paludisme et à songer à des interventions possibles".

Un arbitrage est même parfois possible entre santé et écosystème. Ainsi l'usage du DTT, arme longtemps efficace contre le paludisme, a causé des dégâts important en matière agricole et pose la question du caractère in fine bénéfique de l'opération. D'ailleurs les activités humaines peuvent elles-mêmes être responsables de l'extension du paludisme, ce que montrent les études mettant en relation déforestation et paludisme (Corey et al., 2003).

Un changement des mentalités et des attitudes est-il alors àmême de renverser le cours des événements ? Domarle (2005) évoque pour sa part des habitudes de bon sens : ne plus exposer l'eau de 
pluie dans des citernes en plein Soleil, bien évaluer les symptômes pour ne pas utiliser la quinine de manière abusive, prendre en charge plus rapidement les malades, optimiser les moyens. De même " l'élimination des marécages insalubres, l'introduction de l'hygiène et l'élévation du niveau de vie contribuent beaucoup à l'éradication de la maladie ", (Duriez et Golvan, 1996, p. 419). Est-il alors certain que le paludisme soit une cause de pauvreté ? N'est-ce pas plutôt l'inverse ? Brown (1986) montre par exemple que l'éradication du paludisme n'a pas entraîné le développement. Ce sont les inégalités qui sont en grande partie responsables du "succès" du paludisme (Brown et Inhorn, 1990). Dans leur ouvrage de synthèse, Heggenhougen, Hackethal et Vivek (2003) soutiennent aussi ce point de vue. D'ailleurs la mise en évidence du statut socioéconomique des individus dans l'appréhension de la maladie confirme cette idée. La lutte contre le paludisme n'apparaît alors plus, et contrairement à ce que soutient Sachs, comme la condition nécessaire et suffisante au développement. Il faut envisager une perspective plus large. Il n'est pas certain toutefois que la perspective institutionnelle développée par les protagonistes actuels permette d'y répondre.

\section{Paludisme et institutions dans la littérature récente : une relation tendue et tronquée.}

En se focalisant sur la nature des causes profondes de la croissance et du développement, le débat actuel tente de gommer les liens entre variables. Cela donne alors une vision tronquée du paludisme et de la géographie. Pourtant l'analyse institutionnelle n'est-elle pas a priori adéquate pour comprendre la réalité socioculturelle du paludisme ? En se fondant sur le principe de la bonne gouvernance, la théorie institutionnelle dominante peut-elle offrir une réponse satisfaisante?

\section{1. Les institutions comme cause profonde de la croissance.}

Le rôle des institutions est tellement affirmé au sein des spécialistes de la croissance que Cogneau et Guénard (2002, p. 2) parlent d'un "consensus sur le fait que les écarts de développement proviennent moins de la quantité des ressources productives (capital humain et physique) que des institutions qui organisent l'utilisation de ces ressources ". Ces analyses sont d'ailleurs largement relayées par le Fonds monétaire international ou la Banque mondiale. Les institutions sont alors définies en référence au bon fonctionnement du marché. Rodrik et Subramanian (2003) rappellent que les institutions ont pour fonction de réguler, de stabiliser et de légitimer le marché (et l'ordre marchand). Les institutions seront qualifiées de bonne qualité si elles garantissent ces fonctions. Plusieurs 
indicateurs sont utilisés selon les travaux, comme la modicité du fardeau réglementaire, l'existence d'un Etat de droit, l'absence de corruption, le respect des droits de propriété ou encore les contraintes à l'exécutif. Nous sommes ainsi en présence d'une économie néo-institutionnelle de la croissance dans la mesure où des références à des auteurs plus hétérodoxes comme Commons sont absentes.

La mise en évidence du facteur institutionnel repose toutefois sur une difficulté de taille. Les institutions sont en effet en partie influencées par le niveau de croissance, ce qui rend problématique une relation causale (A. Przeworski, 2003, Rodrik, Subramanian et Trebbi, 2004). Cela prend par exemple la forme d'une demande de protection des droits lorsque la valeur des biens augmente, comme l'a illustré notamment l'Ecole des " property rights " (Demsetz, 1967, Anderson et Hill, 1975). Néanmoins les auteurs parviennent à donner aux institutions un rôle moteur et indépendant notamment en réduisant la géographie à une cause naturelle. Celle-ci est en effet approximée, selon les contributions, par la latitude, les maladies, les journées de froid, l'enclavement, la longueur des côtes, les terres arables, la pluviométrie ou encore les températures maximales (Durlauf, Johnson et Temple, 2004). Or ce point de vue, qui ne sera pas contesté par Sachs et ses collaborateurs, est évidemment réducteur. C'est ce que tente de montrer par exemple la géographie sociale. " [...] Ce qui est premier en géographie sociale, c'est la société et non l'espace. Ce sont les mécanismes, les processus sociaux et sociétaux, le jeu des acteurs publics ou privés de toutes espèces. Si l'on ne cherche pas la société au départ, on ne la trouve pas à l'arrivée [...]. Le moteur, la clé, c'est la société. L'espace est toujours second et si l'on commence par lui, on risque de ne pas comprendre. Mais il est bien évident que l'espace à son tour rend bien compte de tout un jeu de variations qui renforcent ou atténuent les décalages et les tensions entre société locale et société globale ", (Rochefort, 1982, p. 13). Le réductionnisme "naturaliste " de la géographie permet alors aux auteurs de se positionner dans deux camps opposés. A partir du moment où la géographie et le paludisme sont ramenés à leurs attributs naturels il est facile de les opposer aux institutions ${ }^{10}$. Les tests économétriques vont ensuite vérifier le rôle des institutions une fois prise en compte l'action de la géographie. C'est à partir de tels tests que Rodrik et Subramanian (2003, p. 32) peuvent prétendre que "... si la Bolivie faisait l'acquisition des institutions de la qualité de celle de la Corée du sud, son PIB serait proche de 18000 \$ plutôt qu'à son niveau actuel de $2700 \$$ ". Cette relation permet alors d'envisager une proposition plus générale du genre :

10 Comme le souligne Talbot (2005) l'espace est "institué ", ce qui remet en cause l'idée d'un espace naturel. 
SI TEL PAYS ADOPTE LES INSTITUTIONS D'UN PAYS OU LA CROISSANCE EST PLUS FORTE ALORS SA CROISSANCE ATTEINDRA LE MEME NIVEAU.

Un tel résultat est atteint indépendamment du rôle des variables géographiques. Il est alors considéré que ces variables ne jouent qu'indirectement sur le niveau de croissance via les institutions. Nous trouvons une expression particulière de ce principe dans le travail d'Acemoglu, Johnson et Robinson (2001). Ces auteurs ont l'idée de prendre l'exemple des colonies, où les institutions furent crées de toute pièce. Ils distinguent ainsi les colonies de peuplement où de "bonnes" institutions sont mises en place et les colonies d'extraction où règnent des institutions prédatrices. La qualité des institutions dépend notamment du régime de propriété mis en place, l'existence de "property rights " bien définis étant un signe d'efficacité. La mise en place d'un type particulier de colonie et donc d'un certain cadre institutionnel est commandé par le taux de mortalité originel des pays colonisés. En effet les Européens n'étaient pas incités à peupler des lieux hostiles. D'où les institutions prédatrices dans les colonies d'extraction. Nous obtenons ainsi la séquence suivante :

LOCALISATION SPATIALE $\rightarrow$ TAUX DE MORTALITE $\rightarrow$ TYPE DE COLONISATION $\rightarrow$ TYPE D'INSTITUTIONS $\rightarrow$ TAUX DE CROISSANCE.

La force de l'argumentation repose sur l'utilisation d'une variable instrumentale, le taux de mortalité pour expliquer le cadre institutionnel. Dans la mesure où ce taux n'explique plus rien aujourd'hui et que les institutions jouissent d'une certaine pesanteur, celles-ci peuvent bien constituer la cause efficiente de la croissance. "Les colonies où les européens avaient de forts taux de mortalité sont aujourd'hui largement plus pauvres que les colonies où les européens étaient en meilleure santé ".

Ce genre d'analyse, bien qu'intéressant, ne peut avoir de vocation universelle. Au minimum, il y a des écarts importants pour des pays qui n'ont pas fait l'objet de colonisation. Une variable instrumentale, ici le taux de mortalité selon les zones géographiques, ne fait pas une théorie pour Rodrik (2004). Ce dernier reconnaît de même qu'il est "juste de rappeler que la communauté scientifique reste divisée sur la signification de la géographie comme déterminant direct du niveau de revenu ", (p. 5) -sous-entendu que l'approche géographique a des fondements tout aussi valable que l'approche institutionnelle. Ces rappels, par un partisan de l'analyse institutionnelle, ouvrent alors une critique plus systématique de la démarche. C'est Sachs qui ira le plus loin dans cette voie, affirmant que les institutions " ne posent pas problème " ("Institutions don't rule ") et qu'il faut réfuter le " fondamentalisme institutionnel ".

106 Economie et Institutions $-n^{\circ} 8-1^{\text {er }}$ semestre 2006 


\section{2. L'explication paludique, une position de bon sens?}

Sachs considère que le paludisme ne peut être combattu par des réformes institutionnelles et qu'il nécessite plutôt une aide internationale ${ }^{11}$. Cette dernière se justifie d'autant plus que l'insuffisante demande solvable initiale ne va pas inciter les firmes pharmaceutiques à trouver un vaccin. Comme le remarque Arrow (2004), ces dernières ne peuvent pas mettre en place ce que nous pourrions appeler une péréquation où les médicaments sont fournis à prix plus élevés dans les pays riches par rapport aux pays pauvres. Nous comprenons dans ces conditions que Sachs prône une distribution gratuite des moustiquaires en Afrique sub-saharienne. Reste toutefois la question, abordée précédemment, à la fois de la réception culturelle des moustiquaires et des conditions socioéconomiques pouvant interdire le développement.

Sachs conteste fondamentalement la prétention universaliste des approches dites institutionnelles. "En jetant aux oubliettes les explications à facteur unique et en comprenant que la pauvreté procède peut-être autant du paludisme que du taux de change nous serons bien plus créatifs et ouverts d'esprit dans notre approche des pays pauvres ", (Sachs, 2003, p. 41). La géographie joue de manière directe, à travers la prévalence paludique, sur le niveau de croissance. D'ailleurs l'approche défendue par Acemoglu, Johnson et Robinson sur les types de colonies intègre la logique géographique puisque " la plupart des raisons pour lesquelles la géographie semble avoir influencé les choix institutionnels dans la passé (...) s'appliquent encore très probablement aujourd'hui ", (Sachs, 2002, p. 1).

En tout cas la proposition générale à laquelle aboutit la démarche de Sachs est radicalement différente de celle de Rodrik et Subramanian envisagée plus haut :

DANS LES REGIONS OU LE PALUDISME EST ENDEMIQUE, LES INSTITUTIONS NE PEUVENT AUGMENTER DURABLEMENT LA CROISSANCE.

Une telle reconnaissance n'implique dans l'esprit de Sachs aucun déterminisme géographique. "C'est une erreur commune-et

11 Le paludisme initie comme nous l'avons vu un cercle vicieux. Il empêche de dégager des ressources suffisantes de contrôle et limite ainsi les chances de développement de l'Afrique sub-saharienne en particulier. "Les chiffres de la santé publique dans les pays pauvres sont mauvais. L'Afrique subsaharienne a un revenu annuel moyen par habitant de $500 \$$, et si on exclut l'Afrique du Sud avec ses 3000 \$ par habitant, la moyenne tombe juste un peu au-dessus de $300 \$$. Les gouvernements des pays pauvres, même lorsqu'ils ne sont pas financièrement limités par des crises de la dette, sont rarement capables de consacrer $4 \%$ de leur PNB pour la santé publique, et la moyenne est d'environ $1,8 \%$ pour l'Afrique sub-saharienne (en excluant l'Afrique du Sud), (Sachs, 2001, p. 521). Cf. aussi Corey et al., (2003, p. 5). 
un argument peu convainquant-de penser que géographie équivaut à déterminisme. Même si une bonne santé est importante pour le développement, les régions paludéennes ne sont pas toutes condamnées à la pauvreté ", (Sachs, 2003, p. 40). Nous retrouvons alors le débat sur les possibilités ou non d'un développement en situation de pauvreté initiale. Que les pays soient "prédestinés " ou non à avoir de bonnes institutions n'est pas le problème car cela ne dit rien de la capacité d'intervention effective des institutions. Qu'elles soient de bonne ou de mauvaise qualité ne changerait rien. Ce sont les zones de paludisme qui déterminent le statut des institutions. En ce sens la géographie jouirait bien d'un statut central et l'idée d'une prédominance de la géographie serait conceptuellement maintenue dans la mesure où deux types de zones émergent : celles où le paludisme est endémique et celles où il ne l'est pas. Dans le premier type de zone les institutions ne peuvent effectivement pas grand-chose, confortant ainsi l'intuition de Sachs. Dans les autres types de zones, les institutions peuvent bien avoir un rôle direct sur la croissance et sur l'éradication de la pauvreté. Les deux approches peuvent ainsi être réconciliées, les institutions n'expliquant pas tout (zone à paludisme endémique) mais pouvant expliquer dans d'autres cas la pauvreté (zones à paludisme non endémique). En ce sens nous pouvons comprendre que de manière provocatrice, Sachs proclame que les institutions n' "expliquent rien ". Elles n'expliquent pas pourquoi règne un paludisme endémique et comment en sortir.

Quel que soit le bien-fondé de cette analyse nous devons remarquer qu'elle n'a pas toujours été défendue par Sachs. Dans une contribution écrite en collaboration avec Warner, "Sources of Slow Growth in African Economies ", Sachs accorde une place essentielle au facteur institutionnel en face notamment de la géographie ${ }^{12}$. Ce sont alors les réformes économiques structurelles qui expliqueraient la croissance. Sachs et Warner en viennent même à défendre une position qui sera précisément celle de Rodrik et Subramanian concernant la quantification à la fois de la qualité des institutions et de la croissance induite. "Nos estimations suggèrent que même avec ses désavantages naturels, l'Afrique aurait pu avoir une croissance par tête supérieure à $4 \%$ avec les politiques appropriées ", (Sachs et Warner, 1997, p. 361). Dans ce cas il n'existe pas de spécificité subsaharienne et une théorie universelle est possible, avec des contraintes particulières liées par exemple au climat ou à l'enclavement. Bien que de telles contraintes apparaissent

12 Trois groupes de causes sont invoqués : les facteurs naturels (proximité des côtes, abondance de ressources, nature du climat), les facteurs liés à la politique économique (ouverture au commerce international, investissements publics, institutions de marché) et enfin des causes sociodémographiques (espérance de vie, démographie).

108 Economie et Institutions $-n^{\circ} 8-1^{\text {er }}$ semestre 2006 
structurelles en ce qui concerne l'Afrique, elles ne semblent pas compromettre la croissance. Le rôle donné ici aux réformes politiques rend problématique le retournement de perspective opéré par Sachs par la suite et précarise du même coup l'explication paludique.

En distinguant en tout cas pays à paludisme endémique et pays sans paludisme, Sachs propose une démarche au cas par cas, annihilant l'idée d'une théorie universelle. Une telle lecture rejoint alors la réflexion engagée plus haut au sujet de la nature multidimensionnelle du paludisme et n'est pas, comme nous allons le voir, incompatible avec la démarche institutionnelle. Pourronsnous sur cette base réconcilier les approches?

\subsection{Vers un rapprochement des points de vue ?}

La dimension socioculturelle et la complexité implicite du paludisme interdisent de toute évidence une politique "clef en main ". Prenant acte de la complexité du réel, en termes de prise en compte des variables et d'interactions entre elles ${ }^{13}$, Mc Kenzie et Samba (2004) montrent qu'à une politique identique peut correspondre plusieurs résultats. Ces auteurs prennent l'exemple de deux modèles, $A$ et $B$, mettant en relation taux de couverture de la population contre le paludisme (grâce à des moustiquaires, des médicaments ou des vaccins) et réduction de la mortalité. A et $\mathrm{B}$ convergent vers des taux à $100 \%$ en termes de couverture et de réduction de la mortalité. Graphiquement, nous obtenons la figure suivante :

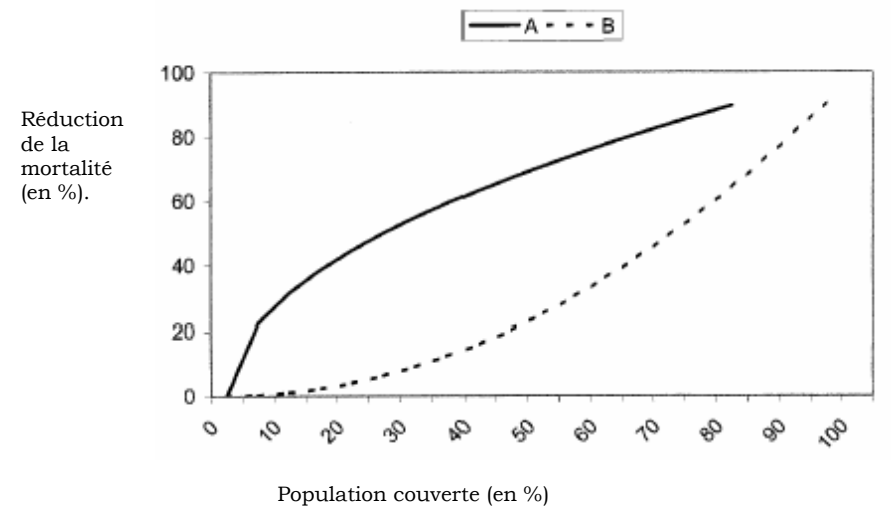

\footnotetext{
13 "Les systèmes biologique, social et autres impliqués sont tellement complexes qu'il n'est même pas possible de définir toutes les variables, et encore moins de donner des prédictions précises sur leurs interactions et résultats généraux dans une situation donnée particulière ", (McKenzie et Samba, 2004, p. 95).
} 
Pour reprendre l'exemple des auteurs, à une réduction du taux de mortalité de $50 \%$ peut correspondre un taux de couverture de la population inférieur à $30 \%$ (modèle A) ou supérieur à $70 \%$ (modèle B). C'est alors la façon d'envisager les variables significatives, la sous-population étudiée à l'origine, etc. qui expliquera la différence. La manière de mener une politique d'aide risque aussi de varier selon les cas. Mc Kenzie et Samba prennent même le soin d'insister sur l'honnêteté et la compétence des chercheurs pouvant effectivement construire des modèles divergents. Malgré tout les auteurs restent optimistes dans la mesure où la modélisation peut se révéler bénéfique comme dans le cadre du Programme de contrôle de l'Onchocerciasis mené en Afrique de l'Ouest.

Les difficultés liées à la phase d'application d'une politique ne sont-elles pas précisément au cœur de la démarche institutionnelle et même géographique ? De ce point de vue des rapprochements sont possibles entre sphères d'étude. L'articulation théorie-pratique constitue déjà un souci majeur pour la géographie au moins telle que l'envisage Lacoste (1981, p. 14). "L'exposé d'un corps de doctrine théorique élaboré à un très haut degré d'abstraction qui cherche à rendre compte de tout le mouvement historique du mode de production capitaliste est nécessaire mais il ne suffit pas pour ceux qui sont durement confrontés, dans leur lutte, aux réalités les plus concrètes. Pour pouvoir agir utilement et élaborer une stratégie et des tactiques efficaces qui tiennent compte du lieu et du moment où se situe leur action, il leur est nécessaire de disposer d'un ensemble de connaissances élaborées à un moindre degré d'abstraction, donc moins généralisables dans le Temps et dans l'Espace ". La démarche institutionnelle, qui consiste au moins dans les intentions, à prendre en compte le monde réel, confirme ce point de vue. Au sein de l'économie néo-institutionnelle, Coase nous paraît révélateur de cette posture, même s'il ne se l'applique pas toujours (Longuépée, 2004). Sa réflexion porte alors sur les solutions aux nuisances en régime de coûts de transaction. "Toutes les solutions ont un coût et il n'y a aucune raison de supposer que la régulation gouvernementale doive être appelée simplement parce que le problème n'est pas bien traité par le marché ou la firme. Des vues satisfaisantes sur la politique ne peuvent venir que d'une étude patiente de la manière dont, en pratique, le marché, les firmes et le gouvernement traitent le problème des nuisances ", (Coase, 1960, p. 18).

Plus récemment les protagonistes du débat sur les causes profondes de la croissance sont également soucieux des spécificités locales. Comme le remarque Rodrik (2003, p. 13), les pays qui ont réussi dans la mise en marche de la croissance sont ceux qui ont combiné des "éléments hétérodoxes avec des hérésies locales". Il 
faut entendre par "éléments hétérodoxes " les pratiques de la bonne gouvernance liées, comme nous l'avons vu précédemment, au respect des droits de propriété, à l'absence de corruption, etc. Il peut exister pour Rodrik plusieurs manières de protéger les droits de propriété. Ainsi la Chine sécurise les investissements privés, notamment étrangers, par un partenariat privé/public. La protection des droits ne repose pas sur l'impossibilité légale d'expropriation, comme dans les pays capitalistes, mais sur le fait que l'Etat, en prélevant une part des bénéfices, n'a aucun intérêt à exproprier. Il s'agit en somme d'un mix entre propriété publique et propriété privée.

La prise en compte des cas particuliers suppose d'établir, comme l'indiquent Hausmann, Rodrik et Velasco (2006), le "bon diagnostic ". Or à des diagnostics différents devront correspondre des réformes institutionnelles différentes. Par conséquent des réformes sur une grande échelle, à l'image des politiques d'ajustement structurel menées il y a encore quelques années par le Fonds monétaire international ou la Banque mondiale, sont pernicieuses. $\mathrm{Au}$ contraire, des "accélérations de croissance " sont possibles à partir de mesures limitées mais ciblées (Hausmann, Pritchett et Rodrik, 2004).

Prétendre toutefois qu'il faut intégrer la dimension locale liée à l'histoire ou la culture ne dit pas encore qu'il est possible de le faire. Comme le montre Williamson (2000), il existe plusieurs niveaux institutionnels renvoyant à des temporalités différentes. A ce titre les institutions informelles sont durables, ont une fréquence de changement de l'ordre du siècle, voir du millénaire et incluent les coutumes, les normes religieuses, etc. Même les institutions formelles telles que les droits de propriété, ont une fréquence de changement relativement faible. Etant donné leur degré d'inertie, les institutions informelles relèvent d'une théorie sociologique. De même selon Pritchett $(2006$, p. 21), citant un économiste dont il préfère taire le nom, "on peut définir les politiques comme ce qui peut être changé à dessein, et les institutions comme ce qui ne peut pas l'être ". Le problème tient très certainement au fait qu'il n'existe toujours pas de théorie unifiée des institutions, ce qui joue évidemment sur l'identification des institutions considérées comme pertinentes. Une telle limite va précisément être exploitée par Bardhan (2005) dans une contribution au titre évocateur: "Les institutions sont essentielles, mais lesquelles "14 ? Il critique notamment le recours à la variable "taux de mortalité des colonisateurs dans le modèle d'Acemoglu, Johnson et Robinson (2001). Il propose de lui substituer la variable "tradition étatique ", mieux à même de rendre " compte des forces historiques majeures qui ont un impact sur la

14 "Institutions Matters, But Which Ones? ". 
structure institutionnelle économique et sociale d'une ex-colonie ", (p. 4).

Plus largement la démarche de Bardhan interroge la question de la variable endogène pertinente. C'est généralement le PIB ou le PNB qui sont retenus. Or, Bardhan montre que les droits accordés aux citoyens sont d'autant plus significatifs que l'on retient un indicateur de développement. Nous pouvons d'ailleurs élargir ce point de vue. En effet malgré une certaine prise de conscience mondiale depuis le rapport Bruntland, la croissance, mesurée par le PIB ou le PNB, demeure l'objectif "idéologique " des pays ${ }^{15}$. Ainsi l'Indicateur de développement humain, censé mesurer le niveau de développement, retient aussi le PIB/habitant, à côté de l'espérance de vie, du taux de scolarisation et du taux d'alphabétisation. Même si les "institutions standard mondiales" permettent effectivement d'accroître le taux de croissance, est-ce vraiment l'objectif le plus souhaitable, et à terme le plus soutenable (et donc le plus réalisable) ? L'épuisement des ressources naturelles, la dégradation de l'environnement ainsi que la nécessité de développement des PED militent à l'extrême en faveur d'une décroissance, au moins dans les pays du nord. Insister par ailleurs comme il est d'usage désormais, sur le développement durable, n'est même pas forcément œuvrer pour le développement. Comme le remarque Brunel (2006), le développement durable risque d'assigner à l'Afrique une "fonction récréative et exotique par un Occident en mal d'authenticité (...) ", (p. 15). Or l'Afrique a avant tout besoin de consolider son marché intérieur en procurant notamment un pouvoir d'achat à ses paysans relativement nombreux plutôt qu'en misant sur des exportations aux revenus aléatoires. "Entre l'éléphant et le paysan, il devient plus rentable pour le fonctionnaire de miser sur le premier, parce qu'il draine des recettes touristiques et cynégétiques autrement plus importantes que la préservation de la dignité et du cadre de vie des populations rurales" (p. 15). En ce sens l'aide internationale préconisée par Sachs doit faire l'objet d'une stratégie vraiment cohérente.

L'enjeu concerne bien la politique de développement à mener au niveau mondial. Ainsi l'action du Fonds monétaire international ou de la Banque mondiale, bien que rénovée selon Rodrik (2004), ne fait toujours pas l'unanimité. Pour Chang (2006) l'analyse des institutions dans la littérature dominante n'est ni plus ni moins qu'une "tentative [pour] surmonter les échecs répétés des politiques orthodoxes dans le monde réel", (pp. 53-54)16. Le marché reste

\footnotetext{
15 Non seulement à travers les contributions académiques mais aussi dans les médias.

16 Hibou (1998) considère pour sa part que la philosophie d'intervention des bailleurs de fonds n'a changé que dans la forme.
}

112 Economie et Institutions $-n^{\circ} 8-1^{\text {er }}$ semestre 2006 
fondamentalement la norme et l'échec des politiques résulte de l'absence des institutions conformes initiales. "En d'autres termes, l'argument institutionnel est mobilisé comme moyen de protéger les principes fondamentaux de la science économique orthodoxe, face à son incapacité à expliquer ce qui se passe dans le monde réel ", (Chang, 2006, p. 54) ${ }^{17}$. Cette dernière critique nous semble intéressante dans la mesure où elle prolonge très exactement la dénonciation par Coase de l' "économie des tableaux noirs ", liée au fait que "lorsque les économistes se rendent compte qu'ils sont incapables d'analyser ce qui se produit dans le monde réel, ils inventent un monde imaginaire qu'ils sont alors capables de manipuler (Coase, 1990, p. 24).

Toutefois comment intégrer ces critiques dans l'exemple particulier du paludisme? Après tout la dimension socioculturelle (dont le paludisme est le vecteur) n'est pas ignorée par de nombreux auteurs. La difficulté à surmonter ici est peut-être davantage épistémologique et interroge les prétentions de la théorie économique. Les institutions informelles ne relèvent-elles pas en effet d'une théorie sociologique comme le suggère Williamson? Plus gravement, pour Brousseau (1999), l'économie néo-institutionnelle, issue des travaux de Coase, North ou Williamson, et dont s'inspirent, rappelons-le, les politiques actuelles, ne peut prétendre à la normativité dont jouit l'économie standard. En n'ayant pas de théorie des prix, elle n'a pas de point de comparaison solide avec lequel orienter la réalité. Aussi se fonde-t-elle sur la supériorité non démontrée des mécanismes marchands et des processus implicites de sélection des règles efficaces.

Nous voyons par conséquent que le débat reste largement ouvert dans la mesure où derrière la nécessité admise par tous de prendre en compte le réel se profile la capacité à le faire et à être efficace. Nul doute en tout cas qu'une analyse conjointe des institutions et du paludisme ne permette d'obtenir des avancées aussi bien au niveau théorique qu'au niveau pratique.

\section{Conclusion}

Partant d'une interrogation sur le statut du paludisme, nous sommes arrivés à celui des institutions. Ceci paraît logique puisque le paludisme revêt une dimension socioculturelle propre à mobiliser la réflexion institutionnelle. Les rapports entre approches

17 Nous retrouvons cette logique chez Zindzingre (2005) qui montre que les institutions informelles sont opposées aux institutions formelles dans l'économie néo-institutionnelle. Ainsi l'échec des politiques de mise en place de droits de propriété serait lié au maintien de règles locales informelles. 
institutionnelle et paludique sont pourtant apparus conflictuels dans le débat. Le problème tient notamment dans le fait que le paludisme constitue un fléau multiforme. Ainsi il oblige à une lecture plurielle que n'intègrent pas les analyses centrées sur la recherche d'un déterminant en dernière instance. Par ailleurs le paludisme induit des moyens considérables pour pouvoir être enrayé. Comment alors imaginer une compensation de facteurs à la Montesquieu où de bonnes institutions viendraient dévier la trajectoire commandée par le climat ? Une telle éventualité impliquerait une capacité endogène à sur-compenser le handicap géographique initial. Or, c'est précisément cette capacité qui manque pour Sachs. Mais une telle reconnaissance ouvre précisément la voie à une analyse institutionnelle. En effet, il existe bien des limites endogènes de nature institutionnelle liées à la compréhension de la maladie. L'exemple des moustiquaires et de leur utilisation en constitue une illustration éclairante. Les moyens financiers doivent s'accompagner d'un changement des mentalités. Si le débat glisse alors vers la question de l'articulation institutions formelles/informelles, un sérieux doute demeure quant à la capacité de l'économie néoinstitutionnelle et de sa transposition dans les institutions de Bretton Woods à offrir une théorie convaincante des institutions. Cette conclusion est atteinte d'ailleurs, quelque soit le niveau de validité de la thèse paludique. En ce sens cette dernière ouvre une réflexion indispensable.

\section{Références bibliographiques}

Acemoglu D. , S. Johnson et J. A. Robinson (2001) : "The Colonial Origins of Comparative Development: An Empirical Investigation ", American Economic Review, 91, pp. 1369-1401.

Acemoglu D. , S. Johnson et J. A. Robinson (2002): "Reversal of Fortune : Geography and Institutions in the Making of the Modern World Income Distribution ", The Quarterly Journal of Economics, 117, pp. 1231-1294.

Anderson T. L. et P. J. Hill (1975): "The Evolution of Property Rights : A Study of The American West ", Journal of Law and Economics, $18, \mathrm{n}^{\circ} 1$, pp. 163-79.

Arrow K. (2004): "Les nouveaux antipaludiques réconcilient la biologie et l'économie ", Finances \& Développement, mars, pp. 20-21.

Audibert M. (2004): "Lutte conter le paludisme: approche économique des obstacles à son contrôle ", Sciences sociales et santé, vol. 22, n 4 , pp. 25-32.

Audibert M. , J. Mathonnat, J. Bokossa et A. Diabagaté (2001) : Système de production rizicole et maladies parasitaires dans l'Afrique de l'Ouest: caractéristiques socio-économiques des ménages agricoles en zone de forêt ivoirienne, CRDI, Etudes et documents.

114 Economie et Institutions $-n^{\circ} 8-1^{\text {er }}$ semestre 2006 
Audibert M. , J. Mathonnat, E. de Roodenbeke, A. Pavy-Latourmy (2004) : "Utilisation des services de santé en Afrique : l'approche communautaire en termes d'offre de services est-elle une réponse ?, Colloque développement durable et santé dans les pays du sud, Lyon, 3 décembre.

Bardhan P. (2005) : Institutions Matter's, But Which One?, Draft, University of California, Berkeley.

Breman J. G. , M. G. Alilio et A. Mills (2004) : "Conquering the Intolerable Burden of Malaria: What's New, What's Needed: A Summary ", American Journal of Tropical Hygiene and Medecine, 71 (Suppl. 2), pp. 1-15.

Brousseau E. (1999) : "Néo-institutionnalisme, prix et normativité ", Economies et Sociétés, série P. E., n²8, pp. 5-30.

Brown P. J. (1986): "Socioeconomic and Demographic Effects of Malaria Eradication: A Comparison of Sri Lanka and Sardinia ", Social Science \& Medicine, 22, pp. 847-859.

Brown P. J. et M. C. Inhorn (1990): "The Anthropology of Infectious Disease ", Annual Review of Anthropology, 19, pp. 89-117.

Brun R. , S. L. Croft, D. A. Fidock, S. Nkawa et P. J. Rosenthal (2004) : "Antimalarial Drug Discovery : Efficacy Model for Compound Screening ", Nature reviews - Drug discovery, Volume 3, june, pp. 509-520, http://www. Nature.com/reviews/drugdisc.

Brunel S. (2006) : Une Afrique en mutation, La Documentation française, Documentation photographique, $n^{\circ} 8048$.

Carstensen K. et E. Gundlach (2005): The Primacy of Institutions Reconsidered : the Effects of Malaria Prevalence in the Empirics of Development, Draft, Kiel Institute for World Economics, august.

Carter R. et K. N. Mendis (2002): "Evolutionary and Historical Aspects of the Burden of Malaria ", Clinical Microbiology Reviews, Vol. $15, \mathrm{n}^{\circ} 4$, pp. 564-594.

Chang H.-J. (2006) : "Sur la relation entre les institutions et le développement économique ", L’Economie politique, n³0, pp. 53-65.

Chevallier J.-J. (1993): Histoire de la pensée politique, Grande Bibliothèque Payot, 1ère édition : 1979.

Claval P. (2003) : Causalité et géographie, L'Harmattan.

Coase R. (1960): "The Problem of Social Cost ", The Journal of Law and Economics, $3, \mathrm{n}^{\circ} 1$, pp. 1-44.

Coase R. (1990): "The Firm, the Market and the Law ", in The Firm, the Market and the Law, The University of Chicago Press, Paperback edition, pp. 1-31.

Cogneau D. et C. Guénard (2002): "Colonisation et institutions ", Dialogue, $n^{\circ} 18$, pp. 2-6.

Corey C. G. , R. A. Kramer, B. C. Murray, S. K. Pattanayak et E. O. Sill (2003) : Malaria, Deforestation and Poverty : A Call for Interdisciplinary Policy, Working paper 03-09, Research Triangle Institute. 
Demsetz H. (1967): "Toward a Theory of Property Rights ", American Economic Review, 57, n², pp. 347-59.

Domarle O. (2005) : " La lutte contre le paludisme ou la lutte contre les paradoxes", e-congrès Scienta communication, Réseau international des Instituts Pasteur, 4-9 avril.

Duriez R. et Y. Golvan (1996) : article "paludisme ", Encyclopédia universalis.

Durlauf S. N. , P. A. Johnson et J. R. W.Temple (2004) : Growth Econometrics, Wassar College Economics Working Paper, $\mathrm{n}^{\circ} 61$.

Elverdan Beth et L. Oberländer (2000) : "Malaria in the United Republic of Tanzania : Cultural Considerations and Health-Seeking Behaviour ", Bulletin of the World Health Organization, 78 (11), pp. 1352-57.

Fainzang S. (2000) : "La maladie, un objet pour l'anthropologie sociale ", Ethnologies comparées, n ${ }^{\circ}$, automne, http://alor.univmontp3.fr/cerce/revue.htlm.

Fonds monétaire international (2003): Perspectives de l'économie mondiale, chapitre 3 : Croissance et institutions.

Gallup J. L. et J. D. Sachs (2001): "The Economic Burden of Malaria ", American Journal of Tropical Medicine and Hygiene, 64 $(1,2)$, pp. 85-96.

Hafid-Martin N. (1999) : Evolution et critique de la théorie des climats à travers le XVIIIè siècle en France. Du déterminisme géographique à la liberté politique, Sito Web Italiano per la Filosifia.

Hamoudi A. A. et J. D. Sachs (1999): The Changing Global Distribution of Malaria : A Review, CID Working paper, $\mathrm{n}^{\circ} 2$, Harvard.

Hausman-Muela S. , R. J. Muela et A. K. Mushi (2000): "The Paradox of the Cost and Affordability of Traditional and Government Health Services in Tanzania ", Health policy and planning, 15(3), pp. 296-302.

Hausmann R. , D. Rodrik et A. Velasco (2006) : "Etablir le bon diagnostic ", Finances et Développement, mars, pp. 12-15.

Hausmann R. , L. Pritchett et D. Rodrik (2003): Growth Accelerations, NBER Working paper series, june, http://www.nber.org/papers/w10566

Heggenhougen H. K., V. Hackethal et P. Vivek (2003): The Behavioural and Social Aspects of Malaria and its Control, World Health Organization on Behalf of the Special Programme for Research and Training in Tropical Disease.

Hibou B. (1998), "Economie politique du discours de la Banque mondiale en Afrique sub-saharienne. Du catéchisme économique au fait (et méfait) missionnaire ", Les études du CERI, n³9, mars.

Institut Pasteur (2005) : La recherche vaccinale à l'Institut Pasteur, mars,

http://www.pasteur.fr/actu/presse/documentation/Paludisme.html

116 Economie et Institutions $-n^{\circ} 8-1^{\text {er }}$ semestre 2006 
Jones C. O. et H. O. Williams (2004) : "The Social Burden of Malaria: What Are We Measuring ", American Journal of Tropical Medicine and Hygiene, vol. 71 (Suppl. 2), pp. 156-62.

Josserand Debacker M. (2000): Paludisme: historique, mythes, croyances et idées reçues, Thèse pour le diplôme d'Etat de docteur en Médecine, Faculté de Médecine de Créteil.

Lacoste H. (1981) : Géographie du sous-développement, 4ème édition, PUF.

Longuépée D. (2004) : "Le réalisme de Coase et les relations droitéconomie. Une analyse critique ", Economie et Institutions, $\mathrm{n}^{\circ} 5,2^{\mathrm{e}}$ semestre, pp. 53-76.

Malaney P. (2003): Micro-economic Approaches to Evaluating the Burden of Malaria, CID Working Paper, $n^{\circ} 99$, Harvard.

Malaney Pia, J. D. Sachs et A. Spielman (2004) : "The malaria gap ", American Journal of Tropical Medicine and Hygiene, 71 (Suppl. 2), pp. 141-46.

Mc Kenzie F. E. et E. M. Samba (2004) : "The Role of Mathematical Modeling in Evidence-Based Malaria Control ", American Journal of Tropical Medicine and Hygiene, 71 (Suppl. 2), pp. 94-96.

Montesquieu C. L. de Secondat (1748) : L'esprit des lois, GF Flammarion, 1979.

North D. C. (1990): Institutions, Institutional Change and Economic Performances, Cambridge University Press.

Office parlementaire d'évaluation des choix scientifiques et technologiques (2005) : Rapport sur le risque épidémique, tome 1.

Pépall J. (2003): Paludisme et agriculture au Kenya, Etudes de cas, CRDI, http://www.crdi.ca/ecohealth.

Przeworski A. (2003) : "The Last Instance : Are Institutions the Primary Cause of Economic Development ? ", Colloque Institutions, Behaviour, and Outcomes, CEBRAP, Sao Paulo, 12-14 mars.

Pritchett L. (2006): "La quête continue ", Finances et Développement, mars, pp. 18-22.

Rochefort R. (1983) : "Réflexions liminaires sur la géographie sociale ", dans Actes du Colloque Géographie Sociale, 14-16 octobre 1982, éd. Daniel Noin, Paris.

Rodrik (2003) : "What Do We Learn From Country Narratives " in In Search of Prosperity, Princeton University Press.

Rodrik D. (2004): Getting Institutions Right, Draft, Harvard University, april.

Rodrik D. et A. Subramanian (2003): "The Primacy of Institutions (and what this does and does not mean), Finance \& Développement, june, pp. 31-34.

Rodrik D. , A. Subramanian et F. Trebbi (2004): "Institutions Rule: The Primacy of Institutions Over Geography and Integration in Economic Development ", Journal of Economic Growth, 9, pp. 131-65. 
Sachs J. D. (2001): "A New Global Commitment to Disease Control in Africa ", Nature medicine, Volume 7, n ${ }^{\circ} 5$, pp. 521-23.

Sachs J. D. (2002) : Institutions Don't Rule : A Refutation of Institutional Fundamentalism, Draft, Earth institute, december.

Sachs J. D. (2003): "Les institutions n'expliquent pas tout ", Finance \& Développement, juin, pp. 38-41.

Sachs J. D. (2006): "Getting Practical in Controlling Malaria ", Project syndicate, december, http://www.project-syndicate.org

Sachs J. D. et A. Warner (1997): "Sources of Slow Growth in African Economies ", Journal of African Economies, Volume 6, n 6, pp. 33576.

Talbot D. (2005) : " Les proximités, entre contrainte et libération de l'action : le cas d'EADS ", Economie et Institutions, $\mathrm{n}^{\circ} 6-7,1^{\text {er }}$ et $2^{\mathrm{e}}$ semestres, pp. 153-180.

Williamson O. E. (2000) : "The New Institutional Economics : Taking Stock, Looking Ahead ", Journal of Economic Literature, Vol. XXXVIII, pp. 595-613.

Zimicki S. (1997) : "La promotion des moustiquaires en Afrique subsaharienne " dans Un Mur contre la malaria, chapitre 4, publications du CRDI.

Zindzingre A. (2005): Institutions and Development: A Theorical Contribution, Institute of social studies, The Hague economic research seminar series.

118 Economie et Institutions $-n^{\circ} 8-1^{\text {er }}$ semestre 2006 